\title{
Is Resting Anterior EEG Alpha Asymmetry a Trait Marker for Depression?
}

\author{
Findings for Healthy Adults and Clinically Depressed Patients
}

\author{
Stefan Debener ${ }^{a}$ André Beauducel ${ }^{a}$ Doreen Nesslera,b Burkhard Brocke ${ }^{a}$ \\ Hubert Heilemann ${ }^{c}$ Jürgen Kayser ${ }^{d}$ \\ a Department of Psychology II, Dresden University of Technology, Dresden, bMax Planck Institute of Cognitive \\ Neuroscience, Leipzig, "State Mental Hospital Arnsdorf, Germany, and dDepartment of Biopsychology, New York \\ State Psychiatric Institute, New York, N.Y., USA
}

\section{Key Words}

Depression - Emotion - Laterality · Anterior brain activation - EEG alpha power · Resting EEG · Temporal stability

\begin{abstract}
Several lines of evidence suggest that asymmetric anterior brain activation is related to affective style, linking left hemisphere activation to positive affect and right hemisphere activation to negative affect. However, previous reports of left frontal hypoactivation in depressed patients were not confirmed in recent studies. This study evaluated additional characteristics of resting EEG alpha (8-13 Hz) asymmetry in 15 clinically depressed patients and 22 healthy adults by recording EEG activity on two separate occasions, 2-4 weeks apart. Across both sessions, group differences in anterior EEG asymmetry were compatible with the original hypothesis. However, groups differed in temporal stability of anterior EEG asymmetry, which was retest reliable in controls but not depressed patients. In contrast, temporal stability of posterior EEG asymmetry was acceptable in both groups. Increased variability of anterior EEG asymmetry may be a characteristic feature for depression, and, if so, this would challenge the notion that anterior EEG alpha asymmetry is a trait marker for depression.
\end{abstract}

Copyright $\odot 2000$ S. Karger AG, Basel

\begin{tabular}{ll}
\hline KARGER & ( ) 2000 S. Karger AG, Basel \\
0302-282X/00/0411-0031\$17.50/0 \\
$\begin{array}{l}\text { Fax +4161306 12 34 } \\
\begin{array}{l}\text { E-Mail karger@karger.ch } \\
\text { www.karger.com }\end{array}\end{array}$ & $\begin{array}{l}\text { Accessible online at: } \\
\text { www.karger.com/journals/nps }\end{array}$
\end{tabular}

\section{Introduction}

A growing body of evidence strongly suggests that the right and left cerebral hemispheres are differentially involved in the regulation and processing of emotion [1]. During the past two decades, anterior electroencephalographic (EEG) alpha power asymmetry, an inverse measure of relative brain activation, has been related to hemispheric specialization of fundamental dimensions of affect, resulting in a model of anterior asymmetry and emotion $[1,2]$. This model postulates an association between individual differences in anterior activation asymmetry and emotion: individuals with a relative stronger left-sided activation are biased toward positive-valenced stimuli and approach-related behavior, whereas those with a relative stronger right-sided activation are biased to negative-valenced stimuli and withdrawal-related behavior. In agreement with this hypothesis is that brain-damaged patients with lesions in left frontal areas also showed symptoms of depression [3, but see also 4]. Because temperament abnormalities in depression are commonly characterized by low positive emotionality, i.e. loss of pleasurable engagement [5], reduced left anterior activation may be related to core symptoms of depression.

Left anterior hypoactivation during rest, as reflected by increased left frontal EEG power in the alpha band (8$13 \mathrm{~Hz}$ ), has been reported in acute depressed [6] as well as

Stefan Debener, Dipl.-Psych.

TU Dresden, Institut für Psychologie II

Mommsenstrasse 13

D-01062 Dresden (Germany)

E-Mail debener@rcs.urz.tu-dresden.de 
remitted patients [7]. In nondepressed individuals, resting EEG alpha asymmetry was found to be stable over time [8-10] and therefore assumed to reflect individual differences. In further agreement with the anterior asymmetry and emotion model is that anterior EEG alpha asymmetry, firstly, predicted affective reactivity [11] and, secondly, showed a relation to individual differences in temperament $[10,12,13]$. Infant studies emphasize the importance of asymmetric anterior brain activation for behavior that is associated with biological features of depression [1]. Hence, functional anterior brain asymmetry has been considered as trait marker for the vulnerability to depression, and left anterior hypoactivation in depression is hypothesized to reflect underactivation of the approach system $[1,2]$.

For several years, brain activation has been explored by means of quantitative EEG, where EEG alpha activity $(8-13 \mathrm{~Hz})$ is considered as an inverse correlate of cerebral activation [14]. Recent imaging evidence seems to support this assumption. Using positron emission tomography (PET), Cook et al. [15] found a significant negative correlation between absolute power in the EEG alpha frequency range and simultaneously recorded $\mathrm{H}^{15}{ }_{2} \mathrm{O}$ PETderived cortical activation [15].

Subclinically depressed college students identified by high scores on the Beck depression inventory (BDI) showed increased left frontal alpha power, which is interpreted as left frontal hypoactivation [16]. Similar relations of BDI scores to anterior EEG asymmetry were also reported for healthy men [13]. Among clinically depressed patients, there is ample evidence of left-sided [17] or left anterior hypoactivation [6,18-21], although there are also several contradictory findings [22-24]. However, studies differ widely in EEG methodology (e.g. recording and epoch length, site of reference electrode), patient diagnosis and symptomatology, and medication status. At least, these methodological differences cause tremendous problems when comparing findings. For example, the internal consistency of EEG alpha power asymmetry is inadequate when based on 1-min recording intervals [25]. As another problem, the studies cited above differ with respect to the particular anterior scalp locations (F3/4, F7/8, T3/4) and the specific recording condition (eyes open vs. eyes closed) for which significant effects were found.

From a theoretical point, empirical evidence according to the hypothesized state independence of left frontal hypoactivation in depression is important, particularly because mood states are suspected to modify resting anterior alpha asymmetry in depression [26]. Only one clinical study reported EEG findings for depressed patients that were repeatedly tested [27]. In this study, left anterior hypoactivation was unchanged in patients with seasonal affective disorder (SAD) after 2 weeks of phototherapy. However, the study's results are based on a small sample size of four, and a generalization from SAD to other depressive subtypes is questionable. Therefore, the study by Allen et al. [27] does not provide an adequate estimate for the temporal stability of anterior asymmetry in depression.

The present study aimed to investigate the temporal stability of anterior EEG alpha power asymmetry in a larger sample of depressed patients and healthy adults. Based on the majority of findings reported in the literature, we hypothesized that left anterior hypoactivation, as indicated by greater left than right alpha power in resting EEG, will be related to depression. Furthermore, anterior EEG asymmetry was predicted to serve as trait index of depression and should consequently be stable over time in both clinically depressed patients and healthy adults.

\section{Method}

\section{Participants}

Depressed inpatients were recruited from the depression ward of the State Mental Hospital Arnsdorf, Germany, so that participation did not affect therapeutic treatment. Diagnosis and clinical state were assessed on separate sessions within a few days of the EEG recording sessions. A structural clinical interview [28] was taken by a trained graduate student, and consensus diagnosis was established together with an experienced psychiatrist (H.H.). Patients were excluded if they had a past history or current symptoms of bipolar affective disorder, organic brain disorder, psychosis or substance abuse. Patients were also excluded if there was a change in medication status during the time of participation, since means of treatment were unaffected by participation in this study.

Patients who received lithium as medication were removed from further analysis, because lithium is known to modify EEG alpha asymmetry [29, 30]. Whether other psychotropic medication systematically alters frontal EEG alpha asymmetry is not known yet. In one study on rhesus monkeys [31], diazepam caused a decrease in power in the 4- to 8-Hz frequency band, most pronounced in the left frontal region. In humans, alterations in EEG alpha asymmetry due to antidepressants were not evident $[32,33]$, nor did experimental variations of serotonergic activity alter EEG alpha asymmetry [34], but this evidence is based on only a few relevant studies. Although several pharmaco-EEG studies analyzed effects of antidepressants, data analysis was typically restricted to midline positions [35]. Therefore, these studies do not provide topographic information. In one recent drug study, the therapeutic effects of the two antidepressants tianeptine and fluvoxamine were associated with a shift in anterior EEG alpha power asymmetry [36] in healthy individuals. As of yet, it is unclear whether psychotropic antidepressants have a normalizing effect with regard to brain activation asymmetry, as assessed by EEG alpha power. Further research is necessary to address this issue.

From the initial sample of 33 retested patients within the course of this study, 18 patients had to be excluded after all clinical informa-

\footnotetext{
$\overline{32} \quad \overline{\text { Neuropsychobiology 2000;41:31-37 }}$
}

Debener/Beauducel/Nessler/Brocke/

Heilemann/Kayser 
tion became available after testing. This report includes only those 15 patients who met all inclusion criteria. According to the International Classification of Diseases [37], 2 patients were diagnosed as having a medium depressive episode ( $\mathrm{F} 32.1$ ), and 13 patients had a severe depressive episode without psychotic symptoms (F32.2), 1 of them also meeting criteria for dysthymia (F34.1). The majority of patients were initially tested after receiving a fixed dosage of psychotropic antidepressant medication for at least 6 days, with most patients being on fixed dosage for a considerably longer period. Patients received trimipramine $(\mathrm{n}=8)$, doxepin $(\mathrm{n}=3)$, amitriptyline $(\mathrm{n}=2)$, or citalopram $(n=1)$. Eleven patients received additional anxiolytic medication (lorazepam). One patient did not receive any psychotropic medication. Hospital staff and interested volunteers were recruited as controls through locally posted advertisements, and screened for current or past history of psychiatric illness or substance abuse. A retest for all participants was scheduled 2-4 weeks after the initial EEG recording session. Handedness was assessed with the Edinburgh Handedness Inventory [38]. Participants were also requested to report their mood on the BDI [39], and the Positive and Negative Affect Scales [40] were assessed immediately after EEG data collection. Demographic and self-report data for both patient and control groups are summarized in table 1 . Among the patients were 10 females and 5 males, the control group consisted of a similar gender ratio of 15 females and 7 males. Although control participants were not selected to match the patients in gender, age, education and handedness, sample characteristics were kept similar to facilitate group comparisons. Whereas group differences in resting EEG alpha power asymmetry are not well known for gender, handedness is suspected to covary with resting brain activation asymmetry [41]. Written informed consent was obtained from all participants.

\section{EEG Data Collection and Quantification}

EEG data were collected from 19 scalp positions during four 2-min periods while participants were resting quietly in a comfortable armchair. Each period of resting EEG was recorded with either eyes open $(\mathrm{O})$ or eyes closed $(\mathrm{C})$. Order of conditions was the same in both EEG sessions, and counterbalanced across participants (i.e. $\mathrm{OCOC}$ or $\mathrm{COCO}$ ).

A Ceegraph AC amplifier system and a stretch lycra cap were used to collect EEG data from 19 scalp locations (10-20 system). Using a forehead ground and linked earlobes (A1-A2) as reference, recordings were taken from 3 midline sites $(\mathrm{Fz}, \mathrm{Cz}, \mathrm{Pz})$ and 8 homologous pairs of electrodes over each hemisphere (Fp1/2, F3/4, F7/8, C3/4, T3/4, T5/6, P3/4, O1/2). EEG impedances were kept below 5,000 $\Omega$ and within $500 \Omega$ at homologous sites, including A1-A2. Vertical electro-oculograms were recorded to facilitate artifact recognition. The EEG signal was band-pass filtered $(0.1-30 \mathrm{~Hz})$ and digitized at $256 \mathrm{~Hz}$. Continuous EEG data were divided offline in 2-second epochs ( $75 \%$ overlap) and rejected if visual inspection indicated eye movements or other recording artifacts. For eyes open, controls produced more artifactfree epochs than patients across recording sessions (controls, mean = 258 , range $71-400$, vs. patients, mean $=137$, range $41-321 ; \mathrm{t}_{(35)}=$ $4.02, \mathrm{p}<0.001)$, but there were no group differences for eyes closed (controls, mean $=326$, range 77-458, vs. patients, mean $=350$, range $153-462 ; \mathrm{t}_{(35)}=-0.70$, n.s.). However, as stated below, internal consistency of EEG alpha power asymmety was highly reliable across conditions in both groups. A Hamming window [9] was then applied over the distal $50 \%$ of each epoch. Absolute spectral power $\left(\mu \mathrm{V}^{2}\right)$ as a function of frequency was derived by means of fast Fourier transformation. Epochs were averaged separately for eyes open and eyes closed
Table 1. Demographic and self-report data

\begin{tabular}{|c|c|c|}
\hline & $\begin{array}{l}\text { Patients } \\
(\mathrm{n}=15)\end{array}$ & $\begin{array}{l}\text { Controls } \\
(\mathrm{n}=22)\end{array}$ \\
\hline Gender, women:men & $10: 5$ & $15: 7$ \\
\hline Age, mean (range) & $48.5(23-64)$ & $45.9(26-64)$ \\
\hline Education, mean \pm SD & $9.4 \pm 1.6^{1}$ & $9.7 \pm 1.9$ \\
\hline Handedness, left:right & $1: 14$ & $3: 19$ \\
\hline \multicolumn{3}{|l|}{ Days between EEG recording } \\
\hline sessions, mean $\pm \mathrm{SD}$ & $22.5 \pm 4.7$ & $21.7 \pm 4.2$ \\
\hline $\mathrm{BDI}^{2}$, mean $\pm \mathrm{SD}$ & $20.8 \pm 11.2^{3}$ & $5.1 \pm 3.7$ \\
\hline \multicolumn{3}{|l|}{ State mood, session 1} \\
\hline Positive affect, mean \pm SD & $2.7 \pm 0.9$ & $2.9 \pm 0.8$ \\
\hline Negative affect, mean \pm SD & $1.5 \pm 0.5$ & $1.1 \pm 0.2$ \\
\hline \multicolumn{3}{|l|}{ State mood, session 2} \\
\hline Positive affect, mean $\pm \mathrm{SD}$ & $2.7 \pm 0.8^{1}$ & $3.0 \pm 0.6$ \\
\hline Negative affect, mean \pm SD & $1.5 \pm 0.5^{1}$ & $1.1 \pm 0.2$ \\
\hline 1 & & \\
\hline $\begin{array}{ll}2 & \text { Groups differed significantly } \\
3 & \mathrm{n}=12\end{array}$ & $=4.8, \mathrm{p}<0$ & (two tailed). \\
\hline
\end{tabular}

condition. Power density values were then $\ln$ transformed to normalize data [42]. Statistical analyses were restricted to power in the EEG alpha band $(8-13 \mathrm{~Hz})$ to limit Type I error probability, and because previous studies on individual differences in anterior activation asymmetry have focused on this frequency range.

\section{Statistical Analysis}

Previous studies involving measures of anterior asymmetry have reported effects at mid-frontal (F3/4), lateral-frontal (F7/8), and anterior-temporal (T3/4) sites (see [24] for a detailed discussion). For these locations, significant parametric intercorrelations of asymmetry measures were obtained in the present data for depressed patients $(r=0.68-0.94)$ and healthy controls $(r=0.69-0.94)$. Therefore, electrode sites were pooled within anterior left $(\mathrm{F} 3, \mathrm{~F} 7, \mathrm{~T} 3)$ and right $(\mathrm{F} 4$, F8, T4) regions to reduce the amount of data [17]. Like previous studies, mid-parietal (P3, P4) sites were included to control for regional effects of EEG alpha power [7, 16].

To examine the hypothesized group differences in anterior alpha asymmetry, data from the two recording sessions were submitted to a $2 \times 2 \times 2 \times 2$ repeated-measures analysis of variance (ANOVA), including session $(1,2)$, hemisphere (left, right), and region (anterior, posterior) as within-subjects factors, and group (patients, controls) as a between-subjects factor. Significant interactions were examined through simple effects to locate the source of interaction (BMDP-4V) [43]. To increase statistical power, condition (eyes open, eyes closed) was not included as factor. Furthermore, an explicit goal of this study was to evaluate psychometric properties of alpha asymmetry for both groups separately, and, as described below, this evaluation involved both recording conditions.

Asymmetry measures were also computed by subtracting left-sided power density from right-sided power density at homologous sites (ln right minus ln left). Higher scores on this asymmetry index are interpreted to reflect greater left-sided activation [10]. Temporal stability (rest-retest reliability) of alpha power asymmetry was examined by 


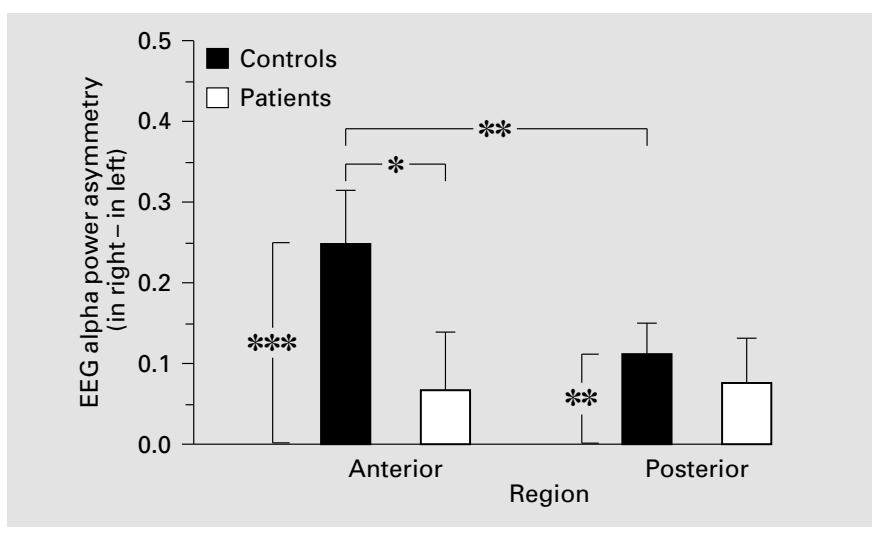

Fig. 1. Mean $( \pm$ SEM $)$ alpha asymmetry (ln right minus $\ln$ left $)$ scores at anterior and posterior sites for healthy controls and depressed patients, averaged over session 1 and session 2. Positive scores indicate more left hemispheric activation. Means were collapsed across mid-frontal (F3, F4), lateral-frontal (F7, F8) and anterior-temporal (T3, T4) sites for the anterior region, and computed for mid-parietal $(\mathrm{P} 3, \mathrm{P} 4)$ sites for the posterior region. The interaction region by group, calculated for the asymmetry indices, was significant, $F(1,35)$ $=4.99, \mathrm{p}=0.032$. Significant mean differences (simple effects, two tailed) are indicated. ${ }^{*} \mathrm{p}<0.1 ; * * \mathrm{p}<0.01 ; * * * \mathrm{p}<0.001$.

computing the Pearson correlations separately for the four indices (anterior: F8-F7, F4-F3, T4-T3; posterior: P4-P3). Internal consistency was determined by Cronbach's $\alpha$ computations of alpha power asymmetry indices, based on two measures for eyes open and two measures for eyes closed conditions. These calculations were done separately for each of the four scalp sites of interest and for each of the two recording sessions. To explore the relationship of anterior asymmetry and mood, correlations of positive affect, negative affect, and overall mood [10] to anterior asymmetry measures were performed.

\section{Results}

The results of the repeated measures ANOVA revealed significant main effects for group, $\mathrm{F}(1,35)=5.49$, $\mathrm{p}=$ 0.02 , hemisphere, $\mathrm{F}(1,35)=11.02, \mathrm{p}=0.002$, region, $\mathrm{F}(1,35)=143.75, \mathrm{p}<0.001$, and session, $\mathrm{F}(1,35)=5.84$, $\mathrm{p}=0.02$. Confirming earlier findings [22], depressed patients (mean $=0.55, \mathrm{SD}=1.05)$ had more alpha power than nondepressed controls (mean $=-0.15, \mathrm{SD}=0.97$ ). As expected, posterior sites (mean $=0.53, \mathrm{SD}=1.09$ ) showed more alpha power than anterior sites (mean $=-0.27, \mathrm{SD}=$ $0.86)$. There was also more alpha power over the right (mean $=0.20, \mathrm{SD}=1.05)$ than left $($ mean $=0.06, \mathrm{SD}=$ 1.07) hemisphere, and at session 2 (mean $=0.18, \mathrm{SD}=$ $1.08)$ than at session 1 (mean $=0.08, \mathrm{SD}=1.04)$.

A significant hemisphere by region interaction, $F(1,35)=4.10, p=0.05$, was indicative of a slightly more pronounced anterior than posterior right-larger-than-left EEG alpha power asymmetry, although simple effects for hemisphere were highly significant for both regions (anterior, $\mathrm{F}(1,35)=10.52, \mathrm{p}=0.003$; posterior, $\mathrm{F}(1,35)=8.73$, $\mathrm{p}=0.006$ ). More importantly, there were regionally dependent differences between groups in left-versus rightsided alpha power, which was supported by a significant hemisphere by region by group interaction, $F(1,35)=$ $4.99, \mathrm{p}<0.032$. Simple interaction effects revealed that the hemisphere by region interaction was significant only for controls, $\mathrm{F}(1,35)=11.19, \mathrm{p}=0.002$, but not for patients, $F(1,35)=0.02$, n.s. A suggested hemisphere by group simple interaction effect emerged only at anterior, $F(1,35)=3.40, p=0.07$, but not at posterior sites, $F(1,35)$ $=0.35$, n.s. Finally, simple hemisphere main effects, calculated for each group and each region, revealed significant greater right than left alpha power only for controls, particularly at anterior sites [anterior, $\mathrm{F}(1,35)=15.96$, $\mathrm{p}<0.001$; posterior, $\mathrm{F}(1,35)=7.76, \mathrm{p}=0.009$ ], but not for patients [anterior, $\mathrm{F}(1,35)=0.82$, n.s.; posterior, $\mathrm{F}(1,35)=2.34$, n.s.). No other significant interaction effects were found in the overall $2 \times 2 \times 2 \times 2$ analysis.

To illustrate the interaction effects involving hemisphere, EEG alpha power asymmetries (ln right minus $\ln$ left) are plotted in figure 1 (interaction effects of two-level factors are statistically equivalent to lower-order effects in an ANOVA on level difference scores). Collapsed across both sessions, controls showed a pronounced left sided activation over the anterior region, i.e. more right than left hemisphere alpha power, and less pronounced leftsided activation over posterior sites, whereas EEG alpha power asymmetry was insignificant in patients for both regions. In contrast to EEG alpha power asymmetry over anterior regions, posterior asymmetry did not differ significantly between patients and controls.

To facilitate comparison with previous research, and because a session main effect indicated overall differences in EEG alpha power between both testing sessions, repeated-measures ANOVA were computed post hoc for each session. These analyses revealed for session 1 suggestive interactions of hemisphere by group, $\mathrm{F}(1,35)=3.56$, $\mathrm{p}=0.07$, and hemisphere by region by group, $\mathrm{F}(1,35)=$ $3.23, p=0.08$. In session 2 , neither of these interactions reached statistical significance [hemisphere by group, $\mathrm{F}(1,35)=0.50$, n.s.; hemisphere by region by group, $\mathrm{F}(1,35)=2.31, \mathrm{p}=0.14] .^{1}$

To address the concern that variations in vigilance may affect group differences in EEG alpha asymmetry, data were reanalyzed by including a factor vigilance, which was operationalized by comparing the first 4 min and the last 4 min of each 8-min recording session. Neither the corresponding five-way 
We further examined internal consistency and temporal stability for each group. Internal consistency computations for each of the four homologous electrode pairs were based on four asymmetry measures during rest, i.e. two measures with eyes open, and two measures with eyes closed. Internal consistencies ranged from 0.84 to 0.98 in the control group, and from 0.87 to 0.98 in the patient group (table 2). These results strongly suggest that EEG alpha power provides a reliable asymmetry measure, irrespective of group, region and session, when averaged over condition (eyes open vs. eyes closed). Excellent reliability in both groups further indicated that group differences in the number of artifact-free epochs (see above) did not bear on reliability.

For nondepressed individuals, temporal stability of EEG alpha power asymmetry (table 2) revealed significant stabilities across the different scalp regions $(r=0.46-$ 0.69 ). For depressed patients, however, a significant testretest stability of $r=0.57$ emerged only for mid-parietal sites; for anterior regions, correlations between recording sessions 2-4 weeks apart were around zero $(r=-0.22$ to 0.09 ) and not significant.

Additional correlational analyses of self-reported positive and negative affect scores did not reveal any systematic relationship to EEG alpha power asymmetry, neither in patients nor in controls.

\section{Discussion}

The present study evaluated regional characteristics and their statistical properties of asymmetric brain activation in depression by assessing EEG alpha power during rest at two different recording sessions. In agreement with prior findings [6,18-21], depressed patients differed from healthy controls in resting anterior EEG asymmetry when data were collapsed across the two recording sessions separated by $2-4$ weeks. Depressed patients failed to show more left than right anterior alpha activity, whereas healthy controls had markedly more right than left anterior alpha activity, i.e. a relative greater activation of left anterior regions. No group differences were found for mid-parietal EEG asymmetry. Although it should clearly be noted that the lack of frontal EEG asymmetry seen in depressed patients did not exactly match previous findings, the direction of group differences in frontal EEG

ANOVA hemisphere $\times$ region $\times$ session $\times$ vigilance $\times$ group nor calculations done separately for each recording session revealed evidence for a significant contribution of the factor vigilance (i.e. for the main effect of vigilance and all interactions involving vigilance, $\mathrm{p}>0.10$ ).
Table 2. Internal consistency and temporal stability of EEG alpha power asymmetry

\begin{tabular}{llc}
\hline & \multicolumn{2}{c}{ Internal consistency } \\
session 1/session 2 & $\begin{array}{c}\text { Temporal } \\
\text { stability }\end{array}$ \\
\hline Patients (n=15) & & \\
$\quad$ Mid-frontal & $0.87 / 0.94$ & 0.09 \\
Lateral-frontal & $0.96 / 0.98$ & -0.22 \\
Anterior-temporal & $0.97 / 0.98$ & -0.05 \\
Mid-parietal & $0.95 / 0.91$ & $0.57^{*}$ \\
\hline Controls (n=22) & & \\
Mid-frontal & $0.94 / 0.95$ & $0.69^{* *}$ \\
Lateral-frontal & $0.97 / 0.98$ & $0.46^{*}$ \\
Anterior-temporal & $0.93 / 0.97$ & $0.53^{*}$ \\
Mid-parietal & $0.84 / 0.95$ & $0.68^{* *}$ \\
\hline
\end{tabular}

Internal consistency based on Cronbach's $\alpha$, temporal stability estimations computed as Pearson's correlations. ${ }^{*} \mathrm{p}<0.05 ;{ }^{* *} \mathrm{p}<$ 0.01 (two tailed).

asymmetry is in line with the theory of a left frontal hypoactivation in depression.

In our study, anterior EEG asymmetry was based on alpha power collapsed across three anterior sites (F3/4, F7/8, T3/4), since initial data analyses did not show any systematic differences in EEG asymmetry for these locations. Furthermore, internal consistency measures of EEG alpha power asymmetry revealed excellent scores of Cronbach's $\alpha$ for both patients and controls, regardless of scalp region. This finding extends previous research of EEG alpha power asymmetry in healthy individuals $[8,9$, 25], by demonstrating that this measure is similarly reliable in clinically depressed patients.

In the present study, EEG alpha asymmetry was solely calculated on the basis of data derived by a linked-earlobes reference, although this reference is critically discussed in the literature [44]. In a previous study [45], we compared linked-earlobes-referenced data to computational Cz-referenced and common-average-referenced data (19-channel recording). The linked-earlobes reference channel comprised less alpha activity in a resting condition, and the corresponding data reflected more appropriately the basic occipitoparietal topography of EEG alpha activity in healthy individuals. However, the results in this report should be interpreted minding the linked earlobes reference, because asymmetry measures derived by different ways of EEG reference may $[6,7,9$, $11]$ or may not $[24,25,45]$ provide similar results. Future work should address this issue with high-density EEG 
recordings to provide higher spatial resolution and the possibility for several additional reference montages.

More importantly, group differences emerged for the analysis of temporal characteristics in anterior EEG asymmetry. Whereas posterior EEG asymmetry was acceptably stable over time in both patients and controls, only controls showed adequate temporal stability of anterior EEG asymmetry. For controls, the level of test-retest stability confirms earlier reports [8-10]. Concerning the insufficient stability of anterior EEG asymmetry in depressed patients, our results differ clearly from one previous report that investigated only four bipolar patients [27]. The group differences in temporal stability of anterior EEG alpha power asymmetry reported here were not biased due to group differences in reliability (internal consistency). It is therefore likely that the temporal instability of anterior EEG alpha power asymmetry provides essential, additional information when characterizing depressed patients in terms of EEG correlates.

Our finding of instable anterior EEG alpha power asymmetry in patients is particularly noteworthy, because this measure has been considered a trait marker for depression [7]. Several reasons may contribute to this instability. First, there is preliminary evidence on an association of state affect and EEG alpha power asymmetry in depression [26]. For this reason, we have explored the relation of the subjects' self-reported mood and anterior EEG asymmetry, but neither in healthy controls nor in depressed patients did we find a significant relation between positive affect, negative affect or a combined measure of mood and anterior EEG asymmetry [for methodology, 10]. Thus, we did not find evidence that state mood is linked to variations in frontal EEG asymmetry, but, of course, we cannot exclude this possibility.

Second, the majority of depressed patients received pharmacological treatment during the course of the study. Although antidepressants are generally not known to alter EEG alpha asymmetry [32,33], a few studies have related therapeutic effects of antidepressants to a shift in anterior EEG alpha power asymmetry $[29,36]$. As stated in the Method section, patients were only included in the study if their medication did not change between testing sessions. However, since patient medication was determined by the course of clinical treatment rather than by the experimental design, the possible influence of antidepressants on anterior EEG alpha asymmetry and its temporal characteristics was uncontrolled in this study. Whether regionally restricted alteration of anterior EEG asymmetry is caused by antidepressant medication is not known yet. Future research may determine whether mood im- provements in clinically depressed patients due to antidepressant medication are accompanied by a shift towards higher left anterior activation.

A third reason for the observed instabile anterior EEG asymmetry in depressed patients may derive from the fact that other clinical symptoms, which often accompany mood disorders, but do not represent core symptoms of depression, could also have an impact on asymmetric brain activation. In particular, different forms of anxiety are associated with different patterns of asymmetric hemispheric activation, thereby raising the issue that a comorbidity of depression and anxiety may conversely affect EEG topography $[17,46]$. It is conceivable, that different clinical symptoms obscure EEG lateralities and vary over time. However, it was beyond the scope of this study to investigate the influence of possible mediator variables like different forms of anxiety.

The temporal instability of anterior EEG alpha power asymmetry in depressed patients is also most likely the cause for the weak relationship of anterior EEG asymmetry to depression that was found in separate analyses for each recording session. If increased variability of anterior EEG asymmetry is indeed a characteristic feature for depression in general, or for some depressive subtypes, this fact may then help to explain recent failures to replicate earlier reports on left frontal hypoactivation in depression. Most previous studies collected EEG data simply only at one recording session $[6,16-21,23$; for a discussion on further possibilities for these failures, see 24]. Although our findings clearly need independent replication, a major goal for future studies should be to unveil circumstances that lead to temporal instability of asymmetric EEG activation seen over anterior brain regions.

To sum up, the findings reported in this study add to the growing body of evidence to show alterations of anterior brain activation in depression. Since anterior EEG asymmetry lacked temporal stability in depressed patients in this study, it appears too premature to consider anterior EEG alpha power asymmetry a trait marker for depression. Further research is needed to uncover the temporal characteristics of anterior EEG asymmetry to clinical features of depression.

\section{Acknowledgments}

We are grateful to R. John, A. Naumann, S. Raabe, D. Schulze and the hospital staff for their help. We also acknowledge the technical support of J. Schäfer, G. Pascher and V. Hantzsch, and thank G. Erdmann for discussions that proved useful in writing this article. $\overline{36}$ Neuropsychobiology 2000;41:31-37
Debener/Beauducel/Nessler/Brocke/ Heilemann/Kayser 


\section{References}

1 Davidson RJ: Cerebral asymmetry, emotion, and affective style; in Davidson RJ, Hugdahl K (eds): Brain Asymmetry. Cambridge, MIT, 1995, pp 361-387.

2 Davidson RJ: Affective style and affective disorders: Perspectives from affective neuroscience. Cogn Emotion 1998;12:307-330.

3 Robinson RG, Downhill JE: Lateralization of psychopathology in response to focal brain injury; in Davidson RJ, Hugdahl K (eds): Brain Asymmetry. Cambridge, MIT, 1995, pp 693711.

4 Borod, JC: Interhemispheric and intrahemispheric control of emotion: A focus on unilateral brain damage. J Consult Clin Psychol 1992;60: 339-348.

5 Fowles DC: Psychophysiology and psychopathology: A motivational approach. Psychophysiology 1987;25:373-391.

6 Henriques JB, Davidson RJ: Left frontal hypoactivation in depression. $\mathrm{J}$ Abnorm Psychol 1991; 100:22-31.

7 Henriques JB, Davidson RJ: Regional brain electrical asymmetries discriminate between previously depressed and normal control subjects. J Abnorm Psychol 1990;99:22-31.

8 Papousek I, Schulter G: Different temporal stability and partial independence of EEG asymmetries from different locations: Implications for laterality research. Int J Neurosci 1998;93: $87-100$.

9 Tomarken AJ, Davidson RJ, Wheeler RE, Kinney L: Psychometric properties of resting anterior EEG asymmetry: Temporal stability and internal consistency. Psychophysiology 1992; 29:576-592.

10 Sutton SK, Davidson RJ: Prefrontal brain asymmetry: A biological substrate of the behavioral approach and inhibition systems. Psychol Sci 1997;8:204-210.

11 Wheeler RE, Davidson RJ, Tomarken AJ: Frontal brain asymmetry and emotional reactivity: A biological substrate of affective style. Psychophysiology 1993;30:82-89.

12 Harmon-Jones E, Allen JJB: Behavioral activation sensitivity and resting frontal EEG asymmetry: Covariation of putative indicators related to risk for mood disorders. J Abnorm Psychol 1997;106:159-163.

13 Jacobs GD, Snyder D: Frontal brain asymmetry predicts affective style in men. Behav Neurosci 1996;110:3-6.

14 Schagass C: Electrical activity in the brain; in Greenfield NS, Sternbach RA (eds): Handbook of Psychophysiology. Hillsdale, Erlbaum, 1972, pp 263-328.

15 Cook IA, O’Hara R, Uijtdehaage SHJ, Mandelkern M, Leuchter AF: Assessing the accuracy of topographic EEG mapping for determining local brain function. Electroencephalogr Clin Neurophysiol 1998;107:408-414.

16 Schaffer CE, Davidson RJ, Sarron C: Frontal and parietal electroencephalogram asymmetry in depressed and nondepressed subjects. Biol Psychiatry 1983;18:753-762.

17 Bruder GE, Fong R, Tenke CE, Leite P, Towey JP, Stewart JE, McGrath PJ, Quitkin FM: Re- gional brain asymmetries in major depression with or without an anxiety disorder: A quantitative electroencephalographic study. Biol Psychiatry $1997 ; 41: 939-948$

18 Kano K, Nakamura M, Matsuoka T, Iida H, Nakajima T: The topographical features of EEGs in patients with affective disorders. Electroencephalogr Clin Neurophysiol 1992;83: 124-129.

19 Roemer RA, Schagass C, Dubin W, Jaffe R, Siegal L: Quantitative EEG in elderly depressives. Brain Topogr 1992;4:285-290.

20 Saletu B, Brandstätter N, Metka M, Stamenkovic M, Anderer P, Semlitsch HV, Heytmanek G, Huber J, Grünberger J, Linzmayer L, Kurz C, Decker K, Binder G, Knogler W, Koll B: Hormonal, syndromal and EEG mapping studies in menopausal syndrome patients with and without depression as compared with controls. Maturitas 1996;23:91-105.

21 Bell IR, Schwartz GE, Hardin EE, Baldwin $\mathrm{CM}$, Kline JP: Differential resting quantitative electroencephalographic alpha patterns in women with environmental chemical intolerance, depressives, and normals. Biol Psychiatry 1998;43:376-388.

22 Pollock VE, Schneider LS: Quantitative, waking EEG research on depression. Biol Psychiatry 1990;27:757-780.

23 Pollock VE, Schneider LS: Topographic quantitative EEG in elderly subjects with major depression. Psychophysiology 1990;27:438444.

24 Reid SA, Duke LM, Allen JJB: Resting frontal electroencephalographic asymmetry in depression: Inconsistencies suggest the need to identify mediating factors. Psychophysiology 1998; 35:389-404.

25 Hagemann D, Naumann E, Becker G, Maier S, Bartussek D: Frontal brain asymmetry and affective style: A conceptual replication. Psychophysiology 1998;35:372-388.

26 Rosenfeld JP, Baehr E, Baehr R, Gotlib ICH, Ranganath C: Preliminary evidence that daily changes in frontal alpha asymmmetry correlate with changes in affect in therapy sessions. Int $\mathrm{J}$ Psychophysiol 1996;23:137-141.

27 Allen JJ, Iacono WG, Depue RA, Arbisi P: Regional EEG asymmetries in bipolar seasonal affective disorder before and after phototherapy. Biol Psychiatry 1993;33:642-646.

28 Pfister H, Wittchen H, Weigel A: Composite International Diagnostic Interview (CIDI). Weinheim, Beltz Test, 1990.

29 Ulrich G, Frick K, Lewinsky M: Lithium and the theoretical concept of 'dynamic restriction': A comparison of the effects on different levels of quantitative EEG analysis. Lithium 1993;4: 33-44.

30 Thau K, Rappelsberger P, Lovrek A, Petsche H, Simhandl C, Topitz A: Effect of lithium on the EEG of healthy males and females. Neuropsychobiology 1988;20:158-163.

31 Davidson RJ, Kalin NH, Shelton SE: Lateralized effects of diazepam on frontal brain electrical asymmetries in rhesus monkeys. Biol Psychiatry 1992;32:438-451.
32 Kwon JS, Youn T, Jung HY: Right hemisphere abnormalities in major depression: Quantitative electroencephalographic findings before and after treatment. J Affective Disord 1996; 40:169-173.

33 Shagass C, Roemer RA, Josiassen RC: Some quantitative EEG findings in unmedicated and medicated depressives. Neuropsychobiology 1988;19:169-175.

34 Knott VJ, Howson AL, Perugini M, Ravindran AV, Young SN: The effect of acute tryptophan depletion and fenfluramine on quantitative EEG and mood in healthy male subjects. Biol Psychiatry, in press.

35 Herrmann WM, Schärer E, Wendt G, DeliniStula A: Pharmaco-EEG profile of levoprotiline. Pharmacopsychiatry 1991;24:206-213.

36 Saletu B, Grünberger J, Anderer P, Linzmayer L, Zyhlarz G: Comparative pharmacodynamic studies with the novel serotonin uptake-enhancing tianeptine and -inhibiting fluvoxamine utilizing EEG mapping and psychometry. $\mathrm{J}$ Neural Transm 1996;103:191-216.

37 Dilling H, Mombour W, Schmidt MH (eds): Internationale Klassifikation psychischer Störungen. ICD-10 Kapitel V (F). Bern, Huber, 1993.

38 Oldfield RC: The assessment and analysis of handedness: The Edinburgh inventory. Neuropsychologia 1971;9:97-112.

39 Hautzinger M, Bailer M, Worall H, Keller F: Beck-Depressions-Inventar (BDI), ed 2, rev. Bern, Huber, 1995.

40 Krohne HW, Egloff B, Kohlmann C, Tausch A: Untersuchungen mit einer deutschsprachigen Version der 'Positive and Negative Affect Schedule' (PANAS). Diagnostica 1996;42: 139-156.

41 Debener S, Nessler D, Friedrich C, Brocke B: Zum Einfluss der Händigkeit auf das SpontanEEG: Alpha-Asymmetrie und interhemisphärische Kohärenz; in Schröger E, Mecklinger A, Widmann A (eds): Experimentelle Psychologie. 41. Tagung experimentell arbeitender Psychologen. Lengerich, Papst Science Publishers, 1999, p 114.

42 Gasser T, Bächer P, Möcks J: Transformations toward the normal distribution of broad band spectral parameters of the EEG. Electroencephalogr Clin Neurophysiol 1982;53:119-124.

43 Dixon WJ (ed): BMDP Statistical Software Manual: To Accompany the 7.0 Software Release. Berkeley, University of California Press, 1992.

44 Miller GA, Lutzenberger W, Elbert T: The linked-reference issue in EEG and ERP recording. J Psychophysiol 1991;5:273-276.

45 Debener S, Beauducel A, Brocke B, Kayser J: Resting anterior EEG alpha asymmetry and affective style: Effects of electrode location and reference (abstract). J Psychophysiol, in press.

46 Heller W, Nitschke JB, Etienne MA, Miller GA: Patterns of regional brain activity differentiate types of anxiety. J Abnorm Psychol 1997;106:376-385. 